\title{
Real Time Traffic Sign Detection Recognition using Adaptive Neuro Fuzzy Inference System
}

\author{
Varun Kumar Singhal \\ Research Scholar \& Assistant professor \\ Patel College of Engineering, Bhopal
}

\author{
Shaik Raheem Pasha \\ Research Scholar \& Software Developer \\ Vertilink Technologies, Hyderabad
}

\begin{abstract}
Traffic Sign Recognition (TSR) framework is a significant part of Intelligent Transport System (ITS) as traffic signs help the drivers to drive all the more securely and proficiently. This paper speaks to another approach for TSR framework where location of traffic sign is done utilizing fuzzy rules based shading division strategy and recognition is refined utilizing Speeded Up Robust Features (SURF) descriptor, prepared by artificial neural network (ANN) classifier. In the identification step, the locale of intrigue (sign region) is divided utilizing an arrangement of fuzzy rules relying upon the tint and immersion estimations of every pixel in the HSV shading space, present prepared on channel undesirable area. At long last the recognition of the traffic sign is executed utilizing ANN classifier upon the preparation of SURF features descriptor. The proposed framework mimicked on disconnected street scene pictures caught under various brightening conditions. The discovery calculation demonstrates a high robustness and the recognition rate is very palatable. The execution of the ANN display is delineated as far as cross entropy, confusion matrix and receiver operating characteristic (ROC) curves. Likewise, exhibitions of some classifier, for example, Support Vector Machine (SVM), Decision Trees, Ensembles Learners (Adaboost) and K Nearest Neighbor (KNN) classifier are surveyed with ANN approach. The recreation comes about represent that recognition utilizing ANN demonstrate is higher than classifiers expressed previously.
\end{abstract}

\section{Keywords}

Traffic Sign Recognition; Fuzzy Rules; Speeded Up Robust Feature; Artificial Neural Network; Confusion matrix; Receiver Operating characteristic Curve, Cross Entropy.

\section{INTRODUCTION}

Traffic sign location and recognition is an imperative research subject that consistently keeps more extensive enthusiasm to the examination in the field of ITS. Since traffic signs show the traffic condition of the street, demonstrate threats and entanglements drivers may experience, offer notices to them, and guide them in route by giving valuable data which makes driving protected and helpful. Because of the huge increment of street vehicles everywhere throughout the world, the quantity of street mishaps has additionally expanded significantly. Among various reasons for mischances, some significant causes are numbness of the street sign, impediment of the street sign and diversion of the drivers. A Driver Assistant System (DAS) like TSR helps the drivers and people on foot to perceive and be ready which help them to be careful from street mischances. Traffic signs don't look same everywhere throughout the world. Henceforth it isn't conceivable to build up a widespread TSR framework. For Bangladeshi traffic signs no significant research works has been finished. Consequently we are emphatically persuaded to do some examination in such manner with a specific end goal to give another measurement in the exploration of TSR in regards to Bangladesh setting. These sign can be characterized as indicated by their shading and shape and both of these decide the significance of traffic signs, as appeared in Table 1.

The most well-known strategies for TSR framework comprise of two principle segments: location and recognition. The creators [1] laid out a thorough viewpoint and study on vision based TSR for ITS featuring diverse calculations of sign location, feature extraction and sign recognition. The greater part of the recognition calculation proposed in the written works utilized shading data for division of the street picture [2, 4, 5, 6, 8,9, 12]. For recognition feature vectors of sectioned locale of intrigue (ROI) is separated. The creators [2] utilized Scale Invariant Feature Transform (SIFT), SURF and Binary Robust Invariant Scalable Key focuses (BRISK) feature descriptor to characterize the street sign and furthermore showed execution examination among these feature vectors. In [9] Histogram Oriented Gradient (HOG) feature vector was utilized to order the street sign. In supervised learning worldview the feature vector is prepared by the classifier demonstrate and these models figure out how to separate the distinctive street signs. Classifiers utilized for recognition of street sign incorporate ANN [8], Adaboost [13, 14], SVM [6, 10, 11, 12] and KNN [2]. The creators [16, 17] created TSR framework in light of content data of the traffic sign. In [18] a novel ROI extraction technique, called High Contrast Extraction was proposed, alongside impediment robust recognition strategy in light of Extended Sparse Presentation Classification (ESRC).

TABLE I: Meaning Of Bangladeshi Road Signs According To Color And Shape

\begin{tabular}{|l|l|l|}
\hline \multicolumn{1}{|c|}{ Color } & \multicolumn{1}{|c|}{ Shape } & \multicolumn{1}{c|}{ Meaning } \\
\hline Red rim & Triangle & Warning \\
\hline Blue rim & Quadrangular & General information \\
\hline Blue & Circle & 'Positive denoting'-mandatory \\
\hline Red rim & Circle & 'Negative denoting'- mandatory \\
\hline
\end{tabular}

The commitment of this paper is to build up a TSR framework for Bangladeshi street signs utilizing fuzzy rules based shading division calculation and recognition by SURF feature descriptor being prepared by artificial neural network classifier. Alongside this, the execution of ANN demonstrate is contrasted and other condition of the earth classifier. The structure of whatever is left of the paper is given as takes after: Section II outlines the proposed framework utilizing a flowchart. Section III speaks to the discovery procedure. After this, the recognition alongside execution investigation of the classifier is explained in section IV. At long last, conclusion and future works are explained in section V.

\section{PROPOSED SYSTEM}

In this paper we propose a traffic sign recognition framework (TSR) for the street signs of Bangladeshi traffic condition. 
The flowchart of calculation for the proposed framework is delineated in Fig.1. The framework comprises of two primary parts: division and recognition. For division the RGB picture is changed over to HSV shading spaces. At that point, the picture is divided utilizing non specific fuzzy rules based picture preparing approach. The tone and immersion estimations of pixel are utilized as information parameters for Fuzzy Inference System (FIS) and the limit shading (red, green, blue, yellow) of the sign as yield. After this, morphological sifting is connected to decrease commotion from the parallel picture. To expel the undesirable locale, territories and perspective proportion parameters are utilized to channel the double picture. At that point the locale of intrigue is removed utilizing jumping box parameters of the divided zone. In the last stage, the recognition of the traffic sign is executed upon formation of SURF features descriptor and preparing of the ANN classifier with scaled conjugate slope back proliferation learning calculation.

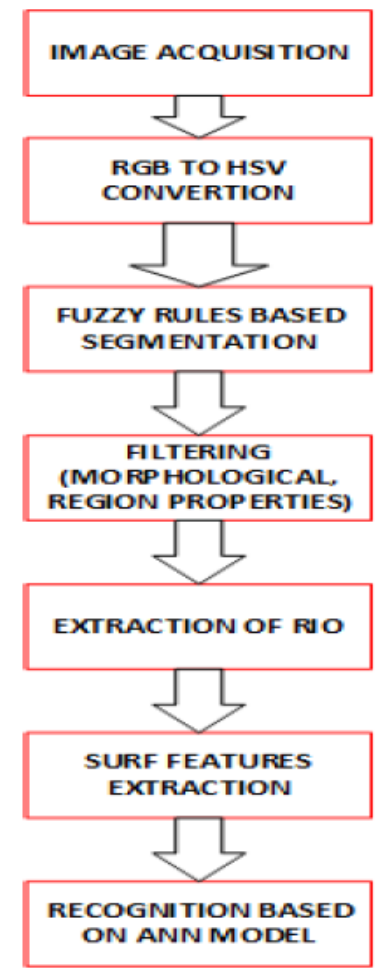

Fig.1. Flow Diagram for the proposed method.

\section{DETECTION}

Location of traffic sign resembles finding the district of intrigue that may contain street signs. Because of the intricate condition of streets and the scenes around them, the location and recognition of street and traffic signs might be dangerous. The shade of the sign blurs with time because of long introduction to daylight. Perceivability is influenced by climate conditions, for example, rain and mists. The shading data is extremely delicate to the varieties of the light conditions, for example, shadows, mists, and the sun. It can be influenced by the illuminant shading (sunlight), enlightenment geometry, and review geometry. The nearness of articles comparative in shading to the street signs in the scene under thought, for example, structures or vehicles. Signs might be discovered muddled, harmed or occulted. Subsequently the recognition of street sign is an uncertain and dubious issue. With the rise of fuzzy rationale ideas, its significance and application in advanced picture handling ended up apparent. Fuzzy set hypothesis is valuable in taking care of different vulnerabilities in PC vision and picture preparing applications. Fuzzy picture preparing experiences three stages: picture fuzzification, derivations and defuzzification.

The fresh contributions to the FIS frameworks are standardized tone and immersion estimations of every pixel. Infuzzification the fresh sources of info are mapped into fuzzy semantic factors to process inside the FLS .The etymological factors that are utilized for the data sources are: red, green, blue, yellow and commotion. The derivation framework has one yield (result). The etymological qualities that are utilized for the yield are: red, green, blue, yellow and dark. With particular enrollment elements of the fuzzy data sources, the Mamdani Inference technique is performed. To demonstrate the procedure with the fuzzy framework, we think about seven rules as takes after:r1 = If (Hue is Red1) and (Sat is Red then (result is Red)

- $\quad$ r2 = If (Hue is Red2 ) and (Sat is Red ) then( result is Red)

- $\quad \mathrm{r} 3=$ If (Hue is Yellow) and (Sat is Yellow) then (result is Yellow)

- $\quad$ r4 = If (Hue is Green) and (Sat is Green) then (result is Green)

- $\quad$ r5 = If (Hue is Blue) and (Sat is Blue) then (result is Blue)

- $\quad$ r6 = If (Hue is Noise1) then (Result is Black)

- $\quad \mathrm{r} 7=$ If(Hue is Noise2) then (Result is Black)

The rules are surmised utilizing AND suggestion technique and the collection of the rules is finished utilizing max min arrangement. At long last the defuzzification is finished utilizing Centroid technique. The participation elements of the Hue and Saturation are inferred in light of instinct and appeared in Fig.2and Fig.3 individually. There are two areas of Hues esteems which are not utilized for street signs are characterized as Noise1 andNoise2. In the event that any of these hues are looked by the fuzzy surmising framework, it reacts by starting a dark pixel. Fig .4 demonstrates the level of enrollment of the outcome (yield variable). There are five part works, one for each shading (red, green, blue, yellow, green). They speak to a specific scope of dim levels in the yield picture which relate to the hues utilized as a part of street signs. The fuzzy surface in figure 5 demonstrates the connection among Hue, Saturation and result. Dim level cutting is utilized to change over the fuzzi prepared picture into parallel picture. Morphological activity with a squared organizing component is performed in the twofold picture to fill little holes and missing limits and to channel numerous start commotion which once in a while spreads on the picture. To get the coveted locale, geometrical features, for example, territory and perspective proportion are figured. Locales that fulfill the properties specified in Table 2 are considered as ROI. The parameters utilized as separating criteria are resolved observationally from the picture datasets. After the division procedure, the sign territory is removed utilizing the bouncing box parameters and this sectioned zone is passed to the recognition advance to determine the SURF features vector and prepare the ANN demonstrate individually. Fig. 6 to 8 delineates the total recognition procedure of street sign of three scenarios.Table 3 presents the location precision of the proposed strategy contrasted and the technique for [9].

\section{TABLE II: Property Used For Filtering}

\begin{tabular}{|l|l|l|}
\hline \multicolumn{1}{|c|}{ Parameter } & \multicolumn{1}{|c|}{ Minimum } & \multicolumn{1}{c|}{ Maximum } \\
\hline Area & 300 & 850 \\
\hline Width & 35 & 53 \\
\hline Height & 35 & 49 \\
\hline
\end{tabular}




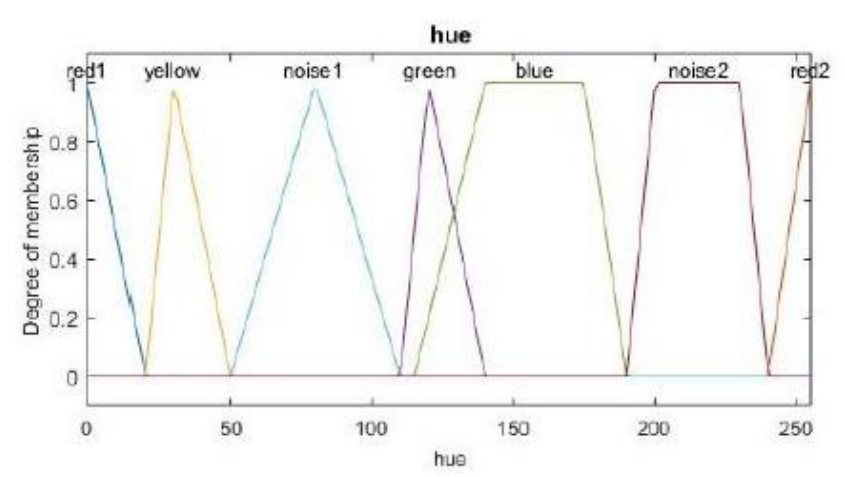

Fig.2. Hue membership functions.

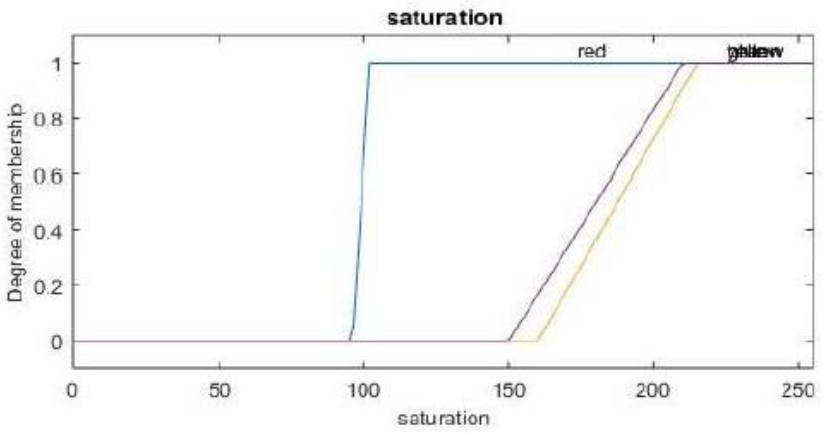

Fig.3. Saturation membership functions.

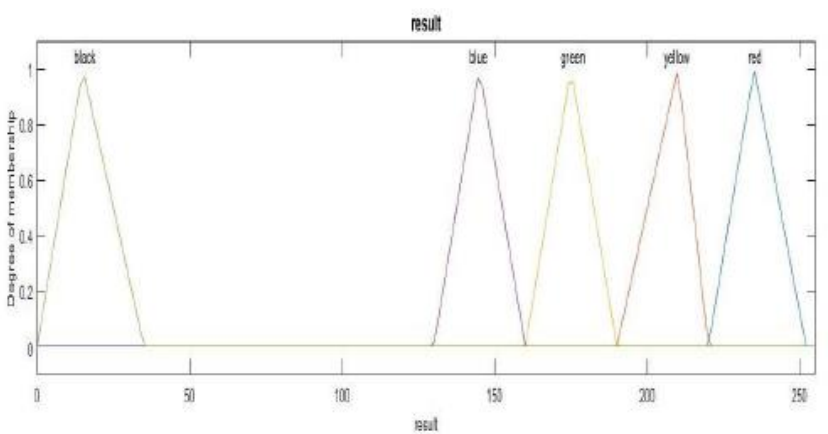

Fig.4. Output membership functions.

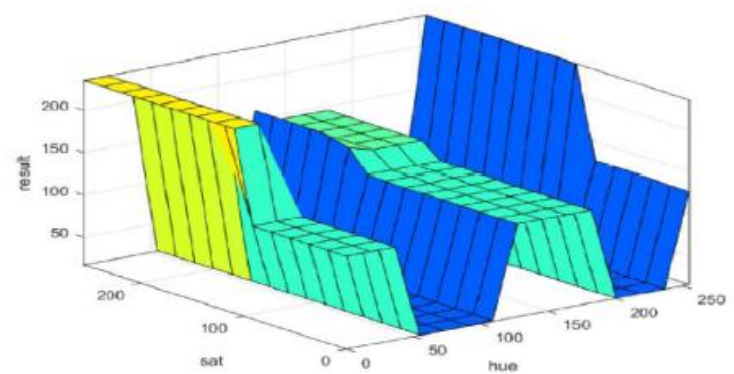

Fig. 5. Fuzzy system surface view.

TABLE III: Comparison Of Detection Accuracy

\begin{tabular}{|l|l|}
\hline \multicolumn{1}{|c|}{ METHOD } & \multicolumn{1}{|c|}{ DETECTION ACCURACY } \\
\hline PROPOSED METHOD & $95 \%$ \\
\hline$[9]$ & $91.73 \%$ \\
\hline
\end{tabular}

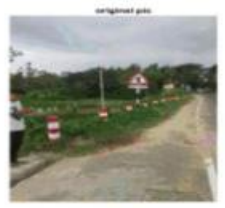

(a)

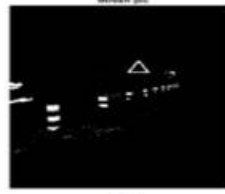

(d)

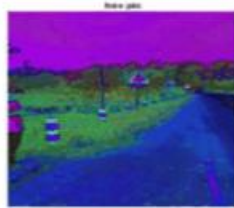

(b)

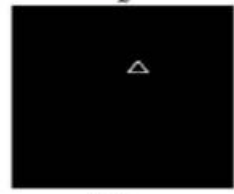

(e)

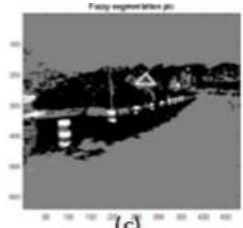

(c)

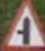

Fig.6. Steps in sign detection a) original image b) HSV image c) fuzzy processed image d) after gray level slicing and e) after filtering and f) ROI extracted using Bounding Box.

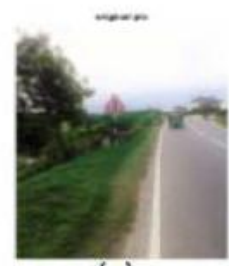

(a)

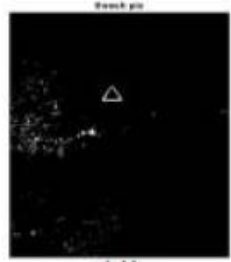

(d)

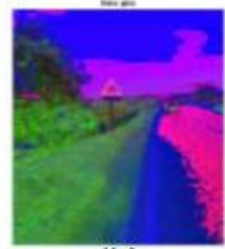

(b)

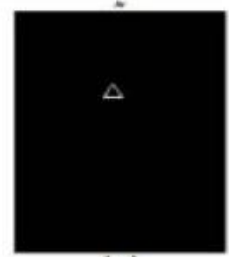

(e)

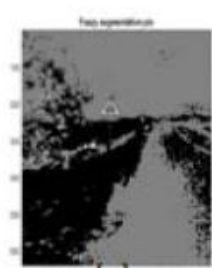

(c) $=\cdots$

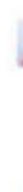

(f)
Fig.7. Steps in sign detection a) original image b) HSV image c) fuzzy processed image d) after gray level slicing and e) after filtering and f) ROI extracted using Bounding Box.
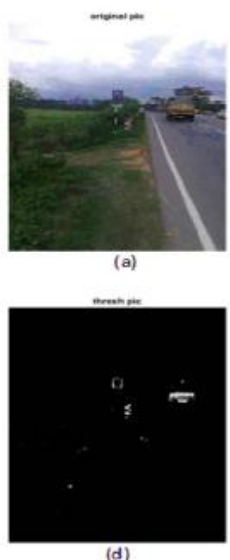

(d)
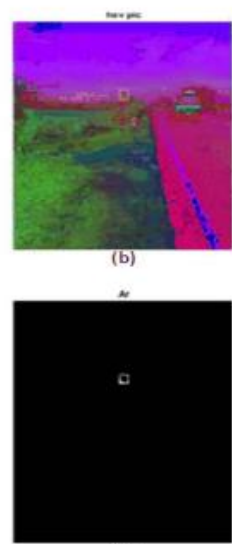

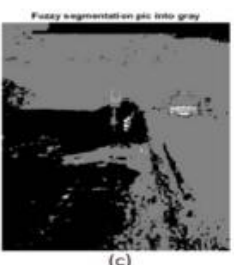

(c)

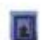

Fig.8. Steps in sign detection a) original image b) HSV image c) fuzzy processed image d) after gray level slicing and e) after filtering and f) RO Iextracted using Bounding Box.

\section{RECOGNITION}

For acknowledgment the features vector is separated from the sectioned sign territory in the dim scale. A genuine test is to productively identify and portray key purposes of an area, with robust portrayal against scale, revolution, and view point 
change. Subsequently the features vector is framed utilizing robust features descriptor (SURF). So as to prepare the ANN display we think about four kinds of traffic signs. Consequently the dataset made for the features extractor speak to the features of four traffic sign.

\subsection{Speeded up Robust Features (SURF)}

The most widely feature descriptor (SIFT) exhibits high computational cost. In 2006, H. Bay et al. proposed a speededup variant of SIFT which was termed as SURF (Speeded up Robust Features) [3]. SIFT computes a 128dimensional features descriptor of neighbors of each key point based on histogram of local gradients On the other hand. SURF depends on sums of Haar wavelet components. Instead of difference of Gaussian, SURF is approximated by laplacian of Gaussian with a box filter. Because of using integral images which allow a very fast computation of box type filters, the computation time has reduced significantly in SURF. The detection of interest point is based on the determinant of Hessian matrix. Based on local maxima of determinant of the Hessian matrix at each location in the image over different scales, the interest point is selected. For each key point an orientation is associated to allow rotation invariance. The orientation is calculated by computing the Haar wavelet response in a circular neighborhood of each key point. The wavelet responses are plotted as points with the strength of horizontal and vertical responses on the two axes. All responses within sliding window are calculated and the leading orientation is selected as the orientation of the key point. The SURF descriptors are calculated by considering a square box filter of size 20 around each key point.

The region around the key point is divided into sub-regions of size $4 \mathrm{x}$ 4and for each block; Haar wavelet responses in horizontal and vertical directions are computed. Each subregion is then represented by a four dimensional descriptor which includes the sum of wavelet responses ( $\mathrm{dx}$ and $\mathrm{dy}$ ) and the sum of the absolute values of $\mathrm{dx}$ and dy. Hence, each subregion has a four-dimensional descriptor vector $\mathbf{v}$ for its underlying

intensity structure $V=\left(\sum d x, \sum d y, \sum|d x|, \sum|d y|\right)$. This results in a descriptor vector for all $4 \times 4$ sub regions of length64. Fig 9 shows the interest point selected by SURF descriptor.
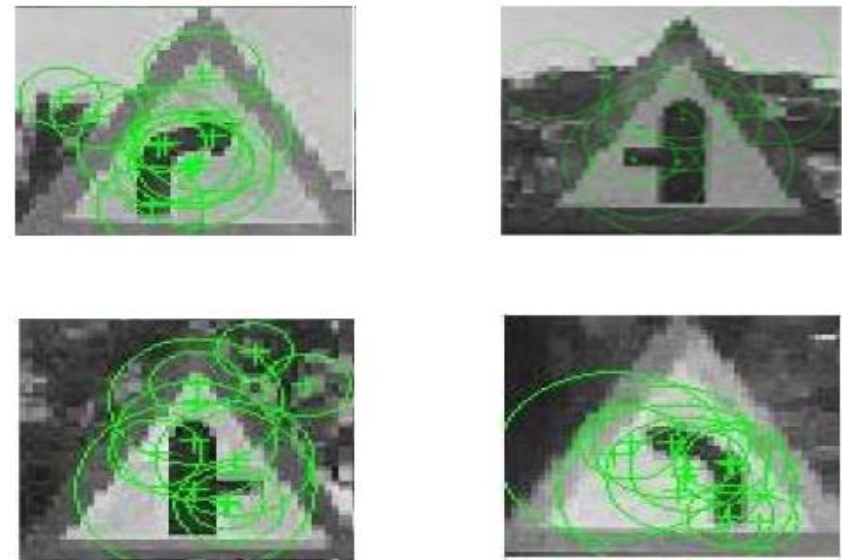

Fig.9. Interest points of SURF features.

\subsection{Dataset}

To prepare the classifier it is critical to make a dataset on every single conceivable condition to stay away from misclassification. However assembling adequate arrangement of genuine information is troublesome. For this we make linguistically street pictures from the genuine picture by evolving splendor, differentiate, including commotion and obscuring. The genuine pictures have been gathered from the diverse high route streets of Bangladesh in various light conditions and distinctive climate conditions. The dataset comprises of four diverse street signs with 50 pictures for every sign and consequently an aggregate of around 200 pictures. A portion of the sign territories (extracted) are presented in Fig. 10.

\subsection{Training and Classification}

ANN is delicate processing apparatus utilized for characterization, information mining (expectation), design acknowledgment, work guess and control framework. A neural network is a hugely parallel conveyed processor made up of straightforward handling units that has a characteristic affinity for putting away experiential information and making it accessible for utilize.
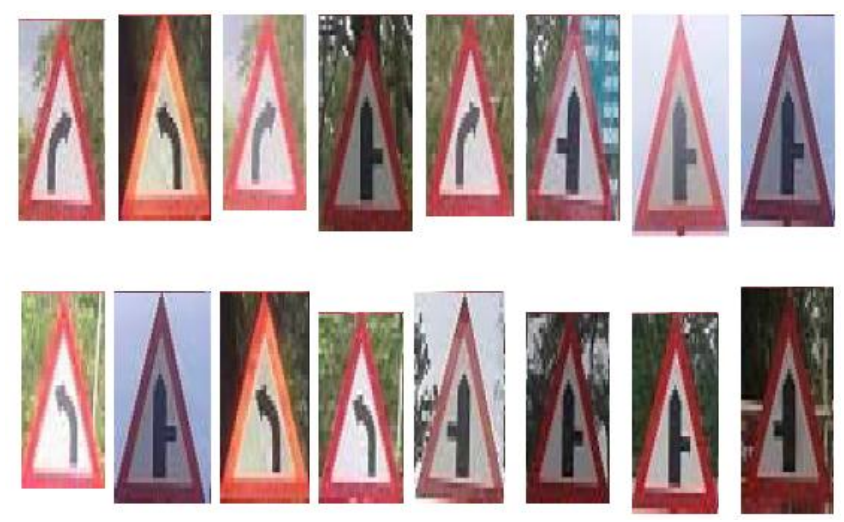

Fig.10. Some examples of extracted road signs.

The fundamental part of ANN is neuron which is basic liner summing intersection interconnected and layered. Every association is weighted by synaptic qualities. The yield of neuron relies upon the synaptic weight. To preparing an ANN implies the change of synaptic weights until want exhibitions is met. An actuation work permits deciding the neurons yields because of weighted aggregates of the data sources. The ANN acknowledgment requires characterizing inputs, kinds of network design, preparing calculation and exchange work. In our works, we utilized multi layered perceptron (MLP) as network topology, nonlinear sigmoid capacity as enactment work and Scaled Conjugate Gradient Back spread calculation as learning calculation. MLP is a bolster forward ANN where information spill out of info layer to yield layer through shrouded layer. MLP networks can learn complex connection amongst information and yield design. The ANN designing procedure requires a lot of information so as to prepare, to approve and to test the network. Here we utilized cross approval methods for better speculation [15].Table 4 shows the parameters and apparatus utilized for preparing the informational collection. 
TABLE IV: Learning Environment And Parameter's

\begin{tabular}{|l|l|}
\hline \multicolumn{1}{|c|}{ Parameters } & \multicolumn{1}{c|}{ Value } \\
\hline Learning tool & $\begin{array}{l}\text { Neural pattern recognition app of } \\
\text { MATLAB 2O15A }\end{array}$ \\
\hline Learning algorithm & $\begin{array}{l}\text { Scaled Conjugate Gradient } \\
\text { Backpropagation algorithm }\end{array}$ \\
\hline $\begin{array}{l}\text { Transfer function } \\
\text { Hidden/Output layer }\end{array}$ & Sigmoid/ Softmax \\
\hline Data Division & $\begin{array}{l}\text { Random(70\% for training, 15\% for } \\
\text { validation and 15\% for testing) }\end{array}$ \\
\hline Network architecture & $\begin{array}{l}\text { Multilayer( 208 x10x4) with one } \\
\text { hidden layer }\end{array}$ \\
\hline Performance & $\begin{array}{l}\text { Confusion matrix , ROC, Cross } \\
\text { Entropy }\end{array}$ \\
\hline No. of Class & Four \\
\hline Features Descriptor & SURF \\
\hline Size of Features vector(Input) & $64 \times 200$ \\
\hline
\end{tabular}

\subsection{Performances Analysis of the ANN Classifier}

In the field of machine taking in a confusion matrix is an estimation that imagines the execution of supervised learning calculation. Every segment of the matrix speaks to the occasions in an anticipated class while each line speaks to the cases in a real class. The confusion matrix of the classifier is portrayed in Fig.11. A Receiver Operating Characteristic (ROC) curve is a graphical plot that shows the execution of a classifier. The curve is made by plotting the True Positive Rate (TPR) against the False Positive Rate (FPR) at different edge settings. The ROC curves of the classifier are delineated in Fig.12.From the confusion matrix it is demonstrated that TPR for all classes are exceptionally palatable. In the experiment of the classifier, TPR for class 1 is $85.7 \%$, for class 2 and 4 it is $100 \%$ and $90 \%$ for class 3.Finally the consolidated TPR is $97.0 \%$ and FPR is $3.0 \%$. The territories under the ROC curves are sign of the execution of the classifier. The greatest territory under the ROC curve is one. It is seen from the ROC curves in Fig. 12 that the regions under ROC curves for all classes are near one. Thus the execution of the classifier is quite palatable. Cross entropy computes a network execution on given targets and yields. Limiting cross entropy brings about great grouping. Fig.13 delineates the cross entropy of the classifier where the best approval is happened at age 11.The table 5 speaks to the near examination of ANN display with other machine students. From the outcome it is watched that the ANN display outflanks than others.

\section{CONCLUSION AND FUTURE WORK}

This paper represents another discovery and acknowledgment calculation with regards to Bangladesh street signs utilizing fuzzy rules based division approach in the enlightenment invariant shading space (HSV) and artificial neural network classifier prepared by robust SURF descriptor. The division comes about represent that it is robust in various light conditions. This feature vector is invariant to scaling, revolution and skewing of the sign because of robust SURF descriptor. The location is created for any traffic sign and acknowledgment is reproduced for four kinds of signs. Here tone and immersion are utilized as information parameter for fuzzy rationale framework. After the division, sifting, for example, morphological examination and geometrical properties are connected.
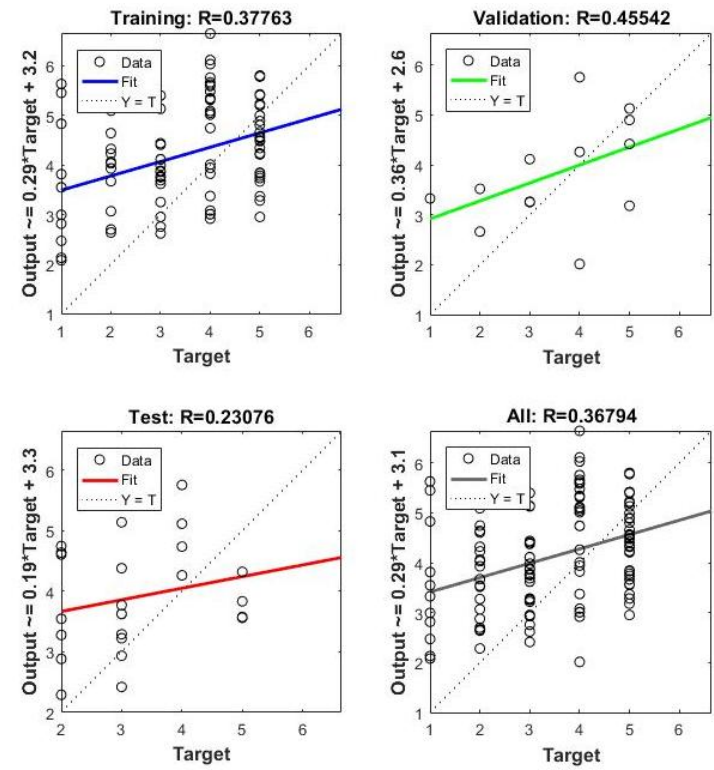

Fig.11. Receiver operating curves of ANN Classifier.

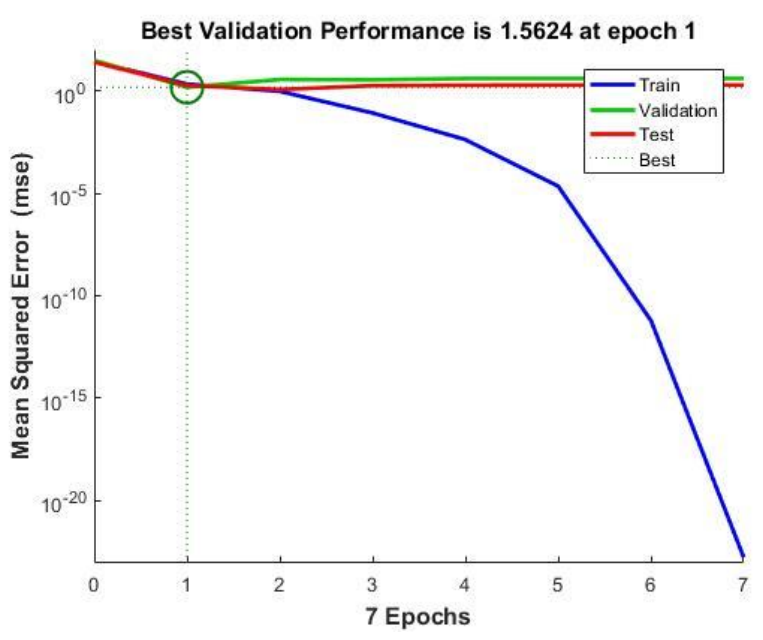

Fig.12. Validation of the ANN classifier.

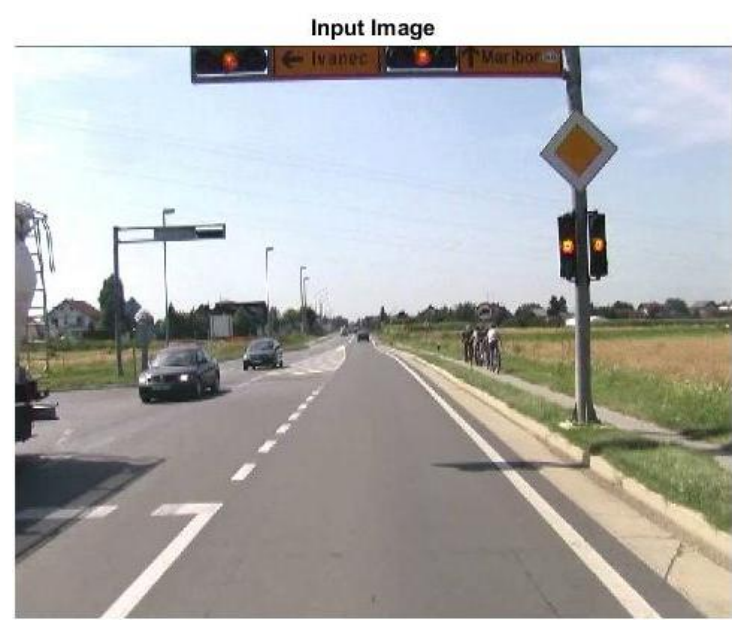

Fig.13. Input Image.

An exact street sign acknowledgment framework with low false positive rate is extremely urgent to contribute more wellbeing and effectiveness. In such manner, the exploratory outcomes speak to that this framework conveys significant 
order rate. From the confusion matrix it is clear that the general right order rate of the ANN classifier utilizing SURF descriptor is $97 \%$. Moreover, a correlation is drawn among the cutting edge classifier where the ANN outflanks in all situations. In future work we plan to consider various difficulties for advance change of the proposed framework.

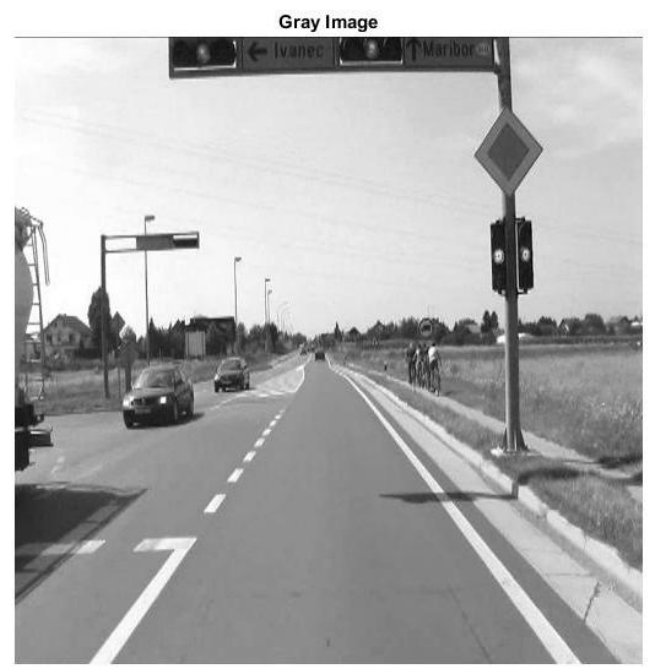

Fig.14. Gray Scale Converted Image

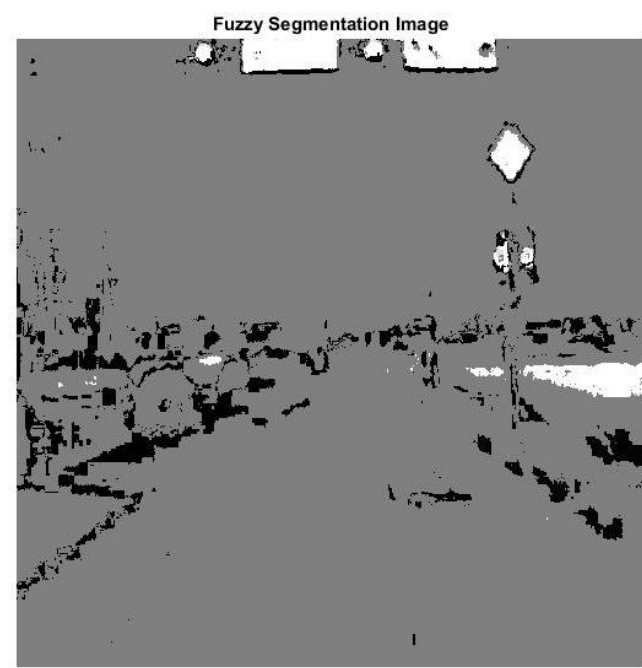

Figure 15. Fuzzy Segmented Image

In such manner we are wanting to address the accompanying issues: to recognize all types of road signs

-to perceive exhibit of

road signs on the scene

-to perceive signs when pictures are taken under night light -to increment the informational collection and

-to build up this framework progressively.

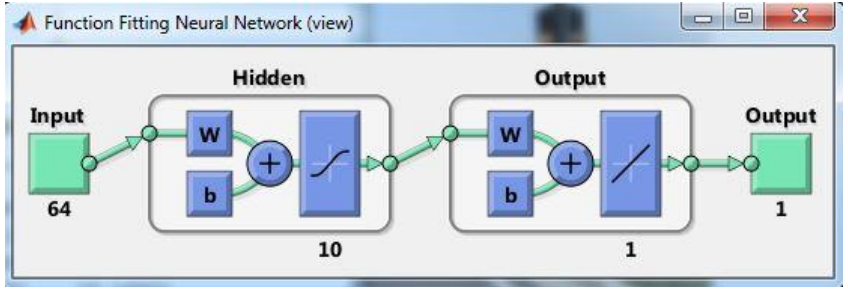

Figure 16. Neural Network Fitting Function

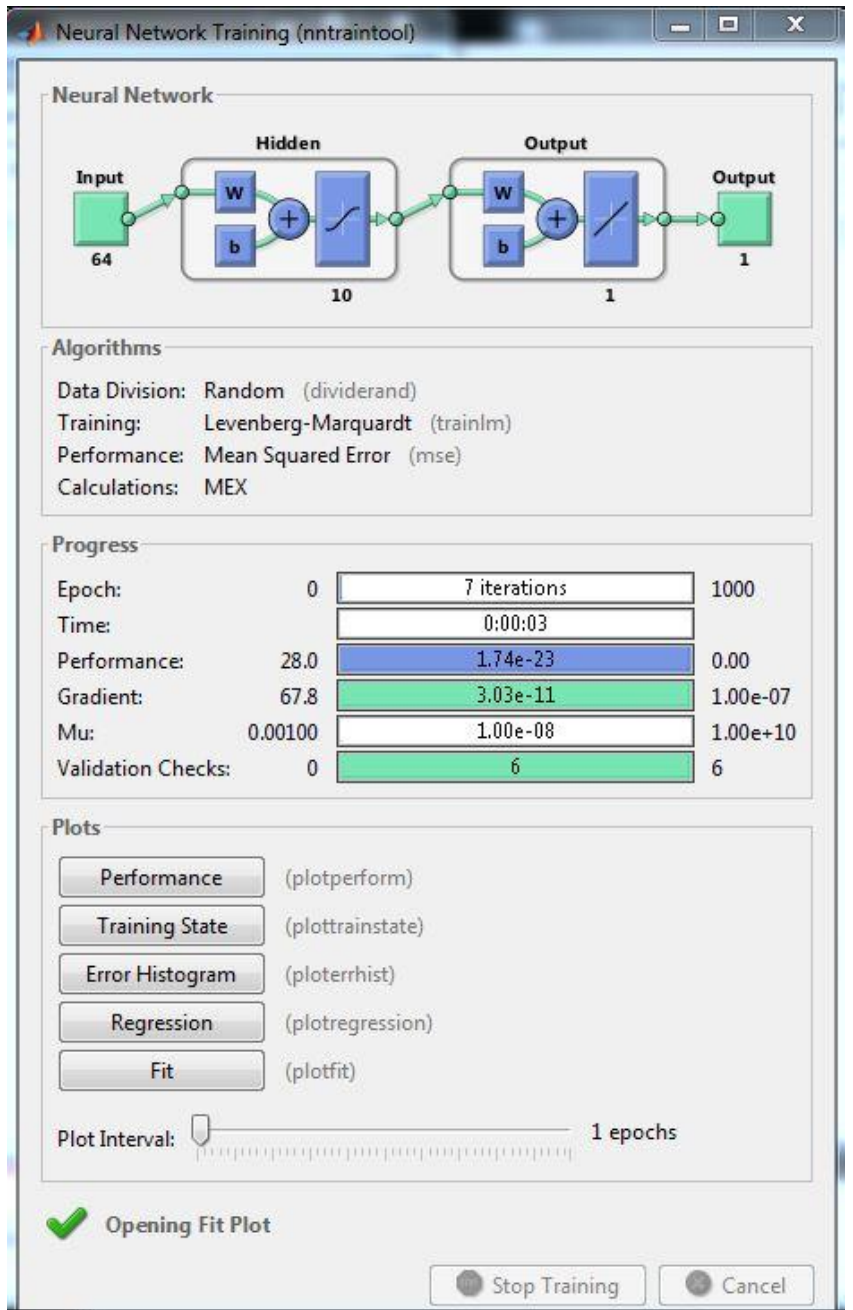

Figure 17. Neural Network Training KNN_SURF KNN_HARRIES

\begin{tabular}{|c|c|c|}
\hline Accuracy & {$[0.7439]$} & {$[0.7724]$} \\
\hline Sensitivity & 1] & 1] \\
\hline Specificity & {$[0.7319]$} & {$[0.7617]$} \\
\hline Precision & {$[0.1486]$} & {$[0.1642]$} \\
\hline Recall & {$\left[\begin{array}{ll}1 & 1\end{array}\right]$} & {$\left[\begin{array}{ll}1 & 1\end{array}\right]$} \\
\hline F-Measure & {$[0.2588]$} & {$[0.2821]$} \\
\hline G-mean & {$[0.8555]$} & {$[0.8728]$} \\
\hline
\end{tabular}

Figure 18. Analysis Parameters 


\section{REFERENCES}

[1] Andreas Møgelmose, Mohan Manubhai Trivedi, and Thomas B. Moeslund,"Vision-Based Traffic Sign Detection and Analysis for Intelligent Driver Assistance Systems: Perspectives and Survey", IEEE Transaction On Intelligent Transportation Systems, Vol. 13, No. 4, December 2012.

[2] Zumra Malik and Imran Siddiqi,"Detection and Recognition of Traffic Signs from Road Scene Image", 12th IEEE International Conference on Frontiers of Information Technology,pp.330-335,2014 .

[3] Bay H , A Ess, T Tuytelaars and L Van Gool, "SURF: Speeded Up Robust Features", Computer Vision and Image Understanding(CVIU).Vol. 110, No. 3, pp. 346$359,2008$.

[4] Yan HanKushal, Virupakshappa \& Erdal Oruklu ,’Robust traffic sign recognition with feature extraction and k-NN classification methods", IEEE International Conference on Electro/Information Technology (EIT),2015 pp. 484 488.

[5] A Broggi, P Cerri, P Medici, P P Porta, G Ghisio, "Real time road signs recognition", IEEE Intelligent Vehicles Symposium, 2007, pp. 981-986.

[6] S. Maldonado-Bascon, S. Lafuente-Arroyo, P. Siegmann, H. Gomez-Moreno, F.J. Acevedo-Rodriguez, "Traffic sign recognition system for inventory purposes", In Intelligent Vehicles Symposium, 2008, pp. 590-595.

[7] Hua Huang, Chao Chen, Yulan Jia and Shuming Tang, "Automatic detection and recognition of circular road sign", In Proc. Of International Conference on Mechtronic and Embedded Systems and Applications, 2008, pp. 626-630.

[8] A. de la Escalera, L. E. Moreno, M. A. Salichs and J. M. Armingol,"Road traffic sign detection and classification", IEEE Transactions on Industrial Electronics, 44(6), 848-859.

[9] Soumenn Chakraborty and Kaushik Deb,” Bangladeshi Road Sign Detection Based on YCbCr color model and DtBs Vector", 1stinternational Conference on Computer and Information Engineering, 26-27 November, 2015.

[10] Jack Greenhalgh and Majid Mirmehdi,"Real-Time Detection and Recognition of Road Traffic Signs", IEEE Transactions on Intelligent Transport Systems, VOL. 13, NO. 4, December 2012.

[11] Saturnino Maldonado-Bascón, Sergio Lafuente-Arroyo, Pedro Gil-Jiménez ,Hilario Gómez-Moren and Francisco López-Ferreras," Road-Sign Detection and Recognition Based on Support Vector Machines", IEEE Transactions on Intelligent Transport Systems, , VOL. 8, NO. 2, June 2007 .

[12] C. G. Kiran, L. V. Prabhu, R. V. Abdu, and K. Rajeev, "Traffic sign detection and pattern recognition using support vector machine", in Proc. ICAPR, 2009, pp. 8790.

[13] C. Bahlmann, Y. Zhu, Visvanathan Ramesh, M. Pellkofer and T.Koehler," A system for traffic sign detection, tracking, and recognition using color, shape, and motion information", In Proceeding of Intelligent Vehicles Symposium. 2005.

\section{AUTHOR's}

Varun Kumar Singhal, has completed B.E (ECE) from Rajiv Gandhi Technical University, Bhopal, M.Tech (VLSI) from Rajiv Gandhi Technical University, Bhopal, Currently he is working as an Assistant Professor of ECE Department in Patel College of Engineering, Bhopal, India.

Shaik Raheem Pasha, has completed B.E (ECE) from Osmania University, Hyderabad. Currently he is working as a Software Developer at Vertilink Technologies, Hyderabad, Telangana, India. 